\title{
Inhibition of CDK4/6 protects against radiation-induced intestinal injury in mice
}

\author{
Liang Wei, ${ }^{1}$ Brian J. Leibowitz, ${ }^{1}$ Xinwei Wang, ${ }^{1}$ Michael Epperly, ${ }^{2}$ Joel Greenberger, ${ }^{2}$ Lin Zhang, ${ }^{3}$ and Jian $\mathrm{Yu}^{1,2}$ \\ 'Department of Pathology, ${ }^{2}$ Department of Radiation Oncology, and ${ }^{3}$ Department of Pharmacology and Chemical Biology, University of Pittsburgh School of Medicine, University of Pittsburgh Cancer \\ Institute, Pittsburgh, Pennsylvania, USA.
}

\begin{abstract}
Radiotherapy causes dose-limiting toxicity and long-term complications in rapidly renewing tissues, including the gastrointestinal tract. Currently, there is no FDA-approved agent for the prevention or treatment of radiation-induced intestinal injury. In this study, we have shown that PD 0332991 (PD), an FDA-approved selective inhibitor of cyclin-dependent kinase 4/6 (CDK4/6), prevents radiation-induced lethal intestinal injury in mice. Treating mice with PD or a structurally distinct CDK4/6 inhibitor prior to radiation blocked proliferation and crypt apoptosis and improved crypt regeneration. PD treatment also enhanced LCR5 ${ }^{+}$stem cell survival and regeneration after radiation. PD was an on-target inhibitor of RB phosphorylation and blocked $\mathrm{C}_{1} / \mathrm{S}$ transition in the intestinal crypts. PD treatment strongly but reversibly inhibited radiation-induced p53 activation, which blocked p53-upregulated modulator of apoptosis-dependent (PUMA-dependent) apoptosis without affecting p21dependent suppression of DNA damage accumulation, with a repair bias toward nonhomologous end joining. Further, deletion of PUMA synergized with PD treatment for even greater intestinal radioprotection. Our results demonstrate that the cell cycle critically regulates the DNA damage response and survival of intestinal stem cells and support the concept that pharmacological quiescence is a potentially highly effective and selective strategy for intestinal radioprotection.
\end{abstract}

\section{Introduction}

Small intestinal epithelium is the fastest-renewing adult tissue, with a turnover rate of 3 to 5 days in mice that is fueled by intestinal stem cells (ISCs) (1, 2). ISCs include actively cycling crypt base columnar cells (CBCs) intermingled with Paneth cells as well as more quiescent cells located slightly above, termed +4 cells. Several genes including the Wnt targets leucine-rich repeat-containing G-protein coupled receptor 5 (Lgr5) and olfactomedin 4 (Olfm4) can mark ISCs in mice (3). Increasing evidence suggests that ISC subpopulations overlap in gene expression profiles and can interconvert upon injury or genetic ablation (3). Genetic ablation of LGR5 cells is well tolerated during hemostasis but profoundly impairs intestinal generation after high-dose radiation (4-6). However, the nature of radioresistant LGR5 cells and their relative contribution to intestinal regeneration remain to be elucidated (7).

Ionizing radiation-induced (IR-induced) gastrointestinal (GI) toxicity is the primary dose-limiting factor in abdominal and pelvic radiotherapy and currently cannot be prevented or treated by any FDA-approved agent $(8,9)$. The single, high-dose total-body irradiation (TBI) model has been used extensively to study IRinduced intestinal injury and regeneration in mice and revealed a key role of the ataxia telangiectasia mutated/p53 (ATM/p53) pathway in regulating intestinal radiosensitivity largely independently of bone marrow injury $(1,7)$. High-dose radiation induces rapid p53/p53-upregulated modulator of apoptosis-dependent (p53/ PUMA-dependent) apoptosis in ISCs within minutes to hours, followed by a delayed nonapoptotic cell death within days during

Conflict of interest: The authors have declared that no conflict of interest exists. Submitted: May 5, 2016; Accepted: August 25, 2016.

Reference information: J Clin Invest. 2016;126(11):4076-4087. doi:10.1172/JCI88410. crypt generation $(10,11)$. Loss of p53 or p21 exacerbates the nonapoptotic response, with elevated DNA damage when the early apoptotic response is either blocked or unaffected (12-14).

Cell-cycle progression is driven by the combination of cyclin-dependent kinases (CDKs) and their cyclin partners. The CDK4/6/cyclin D complex is critical to the $G_{1} / S$ phase transition and is responsible for phosphorylation of the retinoblastoma (RB) protein, the activation of the E2F family of transcription factors, and the subsequent synthesis of factors necessary for DNA replication (15). Therefore, the catalytic activity of CDK $4 / 6$ regulates the commitment to cell division, which is hyperactivated in many human cancers as a result of alternations in cyclins, CDKs, or upand downstream regulators such as p53 and RB (16). As a result, small-molecule, selective CDK4/6 inhibitors (CDKi) have been developed in recent years as potential anticancer drugs, including orally bioavailable PD 0332991 (palbociclib, referred to hereafter as PD) and LEE011 (LEE). PD suppresses the growth of xenografts via an RB-dependent mechanism (17) and received accelerated FDA approval in 2015 for the treatment of estrogen receptor-positive (ER-positive) and human epidermal growth factor receptor 2negative (HER2-negative) breast cancer. However, the effects of CDKi on normal tissues are not well studied.

Radiation induces DNA double-strand breaks (DSBs) and rapidly activates the $G_{1} / S$ checkpoint via ATM- and p53-dependent mechanisms in normal cells $(18,19)$. Conversely, elegant studies in yeast and mammalian cells demonstrate that the induction of $G_{1}$ arrest or genetic ablation of CDKs suppresses radiosensitivity and influences the DNA damage response (DDR) and the choice of DNA repair pathways, while the in vivo significance and underlying mechanisms remain unclear (reviewed in ref. 20). We hypothesize that pharmacologic inhibition of the $G_{1} / S$ transition 
A

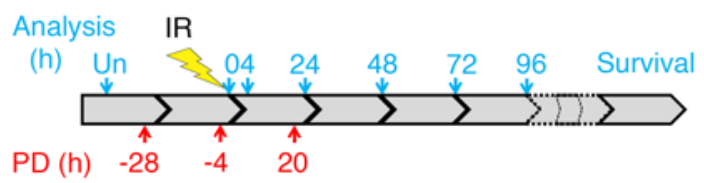

C

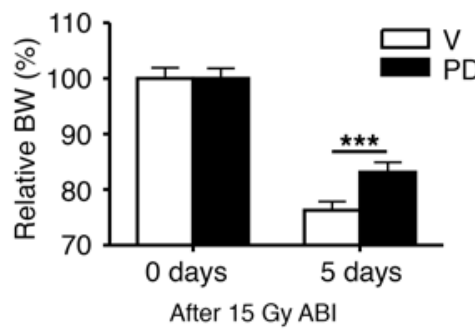

D

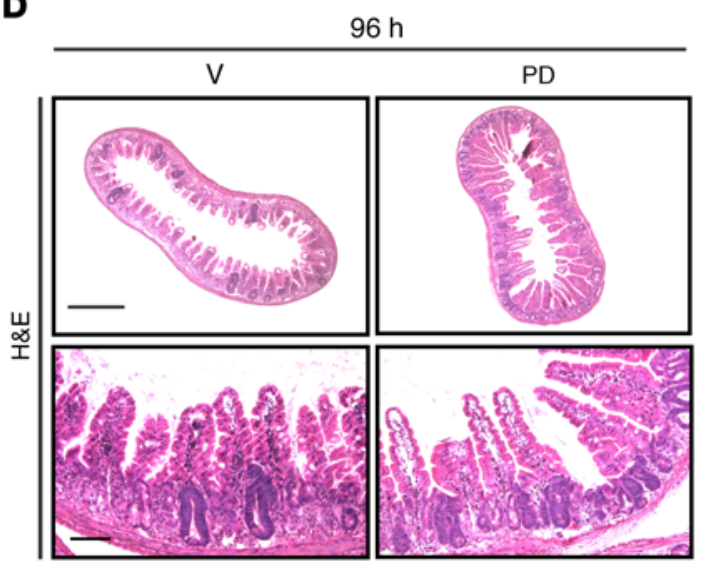

G

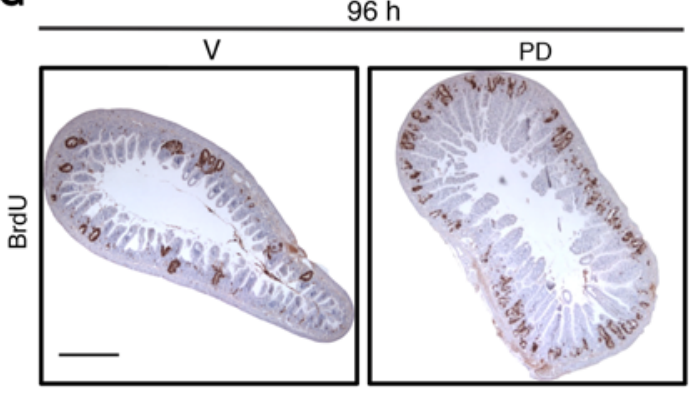

B

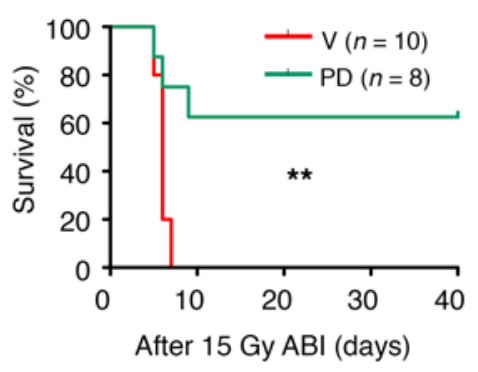

$\mathbf{E}$

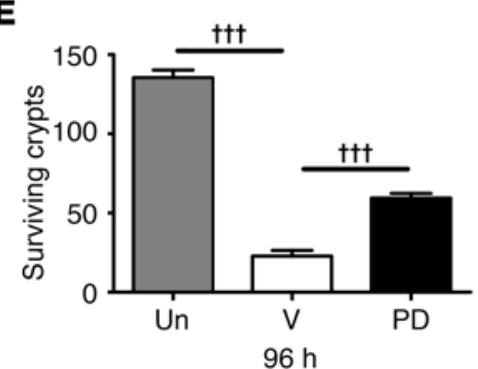

$\mathbf{F}$

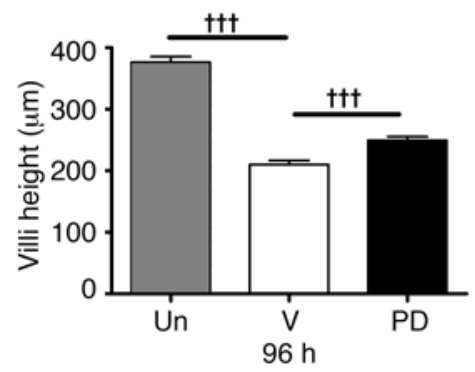

H

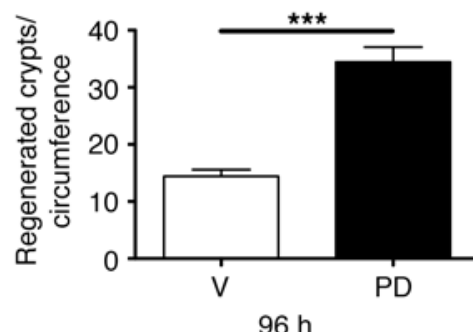

Figure 1. PD prevents IR-induced lethal GI injury. Mice were pretreated with vehicle (V) or PD and subjected to 15 Gy ABI or TBI. (A) Schematics of PD and radiation dosing and analyses at various time points. Un, untreated. (B) Kaplan-Meier survival curve of mice subjected to $A B I$. ${ }^{* *} P=0.0035$, by logrank test. (C) Relative BW on day 5 after $A B I$ compared with $B W$ on day $0 . N=10$ (V) or 8 (PD). (D) Representative images of $\mathrm{H} \& \mathrm{E}$-stained intestinal sections with the indicated treatment at 96 hours. Scale bars: $500 \mu \mathrm{m}$ (top) and $100 \mu \mathrm{m}$ (bottom). (E) Quantitation of surviving crypts 96 hours after TBI. (F) Villus height at 96 hours. (C) Regenerated crypts marked by BrdU IHC in a cross section at 96 hours. Scale bar: $500 \mu \mathrm{m}$. (H) Quantification of regenerated crypts per circumference. (E, $\mathbf{F}$, and $\mathbf{H})$ Values represent the mean $\pm \mathrm{SEM} ; N=3$ mice in each group. ${ }^{t+} P<0.001$, vehicle versus untreated or PD-treated, by 1-way ANOVA followed by Tukey's multiple comparisons test; ${ }^{* *} P<0.001$ vehicle versus $P D$ treatment, by unpaired, 2-tailed Student's $t$ test. prior to radiation will protect the GI epithelium by modulating the DDR. In this study, we discovered that small-molecule CDK4/6 inhibitors potently protect against radiation-induced lethal GI injury by modulating p53 and DNA repair pathways. Our data provide insights into the regulation of the DDR by the cell cycle and potential pharmacological approaches for the selective protection of normal intestinal epithelium and stem cells.

\section{Results}

PD potently protects against IR-induced lethal GI injury. To determine the effect of PD on radiation-induced lethal GI injury, we first monitored the survival of mice after $15 \mathrm{~Gy}$ abdominal irradiation (ABI). The compound was given twice before ( 28 hours and 4 hours) and once after (20 hours) radiation (Figure 1A and Supple- mental Figure 1A; supplemental material available online with this article; doi:10.1172/JCI88410DS1), a schedule chosen empirically so that the last dose would be administered prior to resumption of crypt proliferation, around 24 hours $(1,11,14)$. All vehicle-treated mice died 5-7 days after treatment and had an average weight loss of $24 \%$ by day 5 (Figure 1, B and C), which is characteristic of lethal GI injury. In contrast, $60 \%$ of mice in the PD-treated group survived at least 40 days after treatment, with a significant reduction in weight by day 5 that was restored within 10 days to their preABI weight (Figure 1, B and C, and data not shown). The survival rate of mice after $15 \mathrm{~Gy} \mathrm{ABI}$ or TBI is similar and independent of the $\mathrm{BM}$ injury observed $(21,22)$. However, the ABI model results in uneven GI injury, thus precluding quantitative histological or molecular analysis. Using the well-characterized TBI model (1, 
A

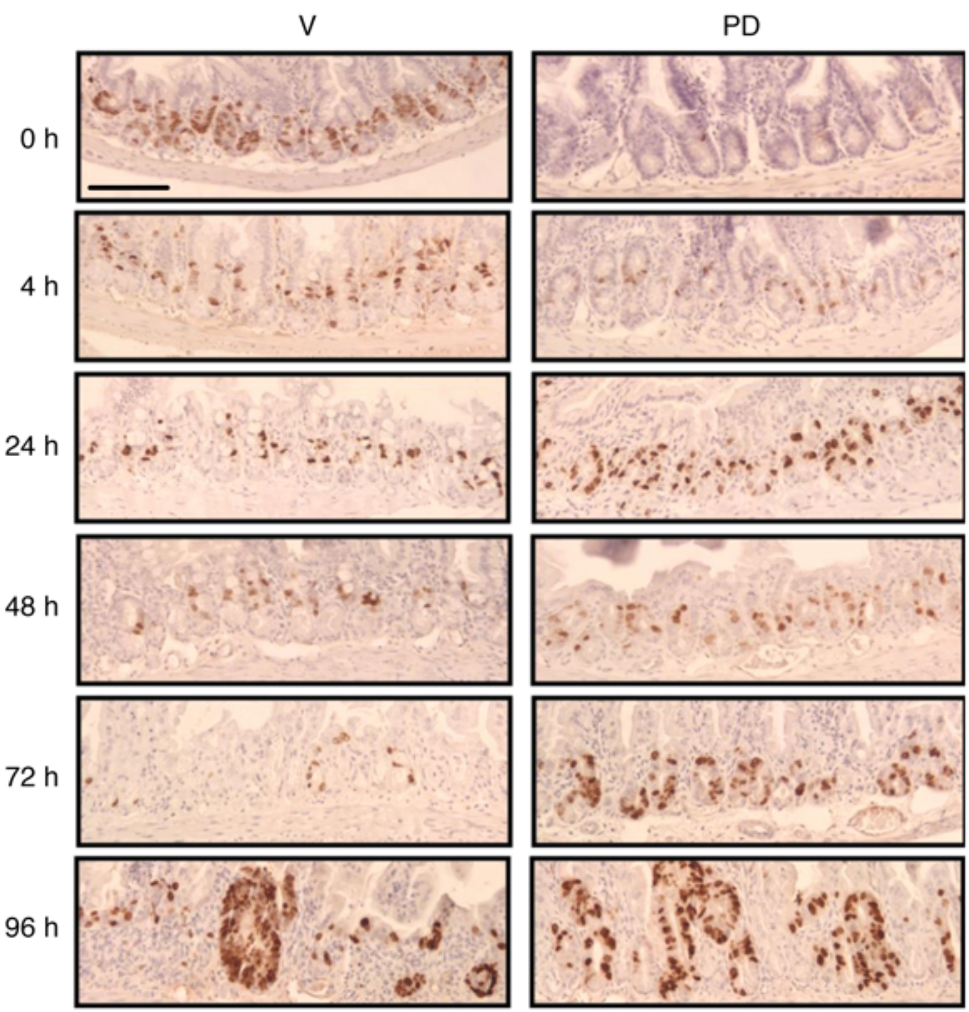

BrdU

B

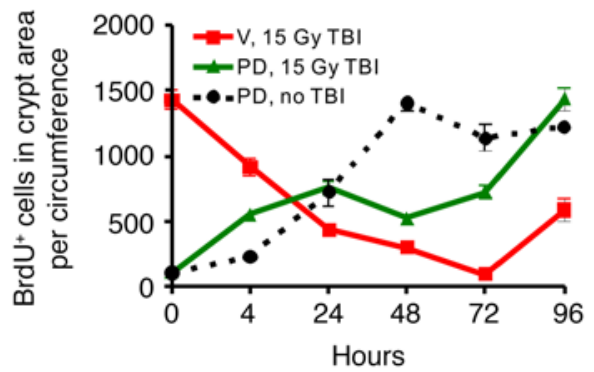

D

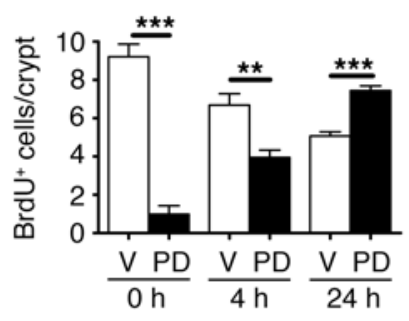

C
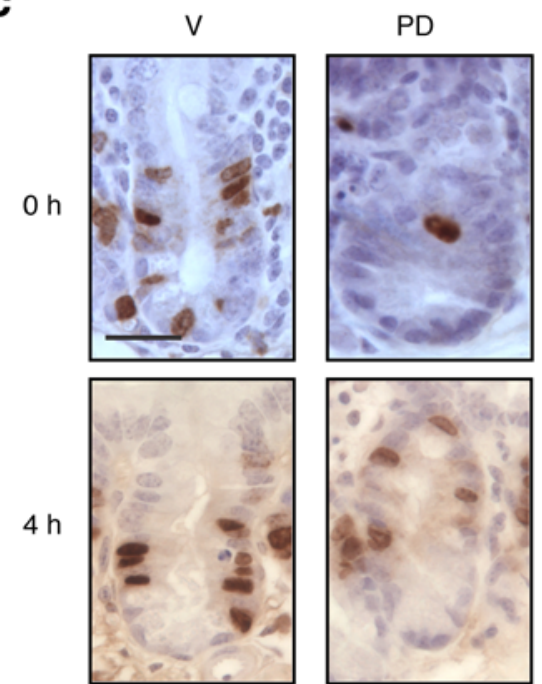

$24 \mathrm{~h}$
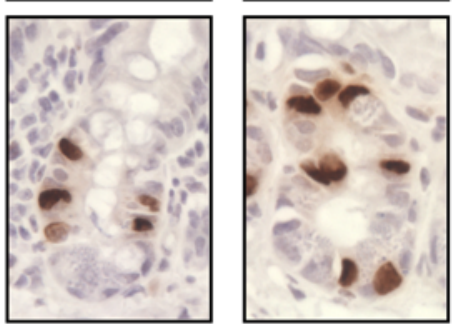

BrdU

E

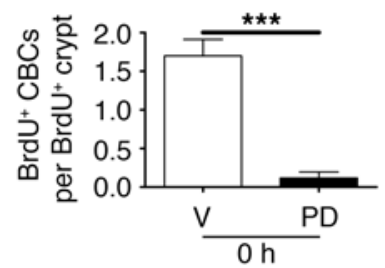

$\mathbf{F}$

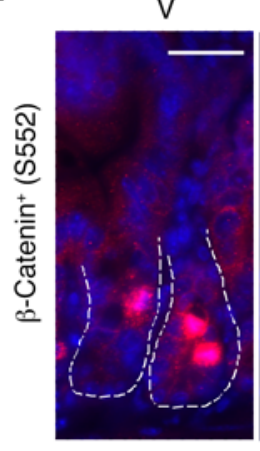

PD

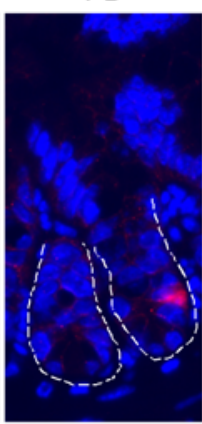

$24 \mathrm{~h}$
G

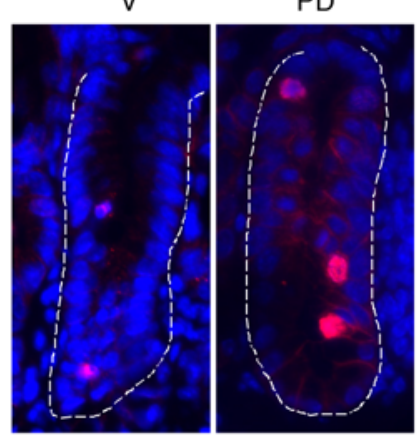

$96 \mathrm{~h}$

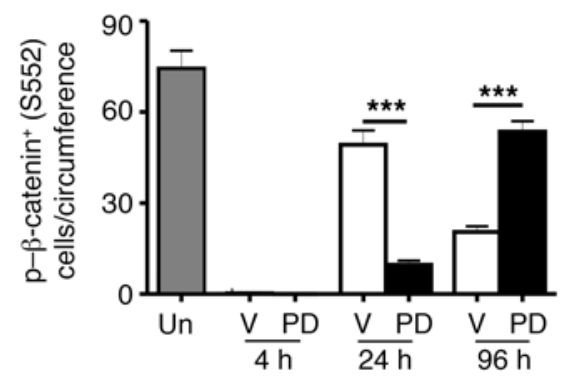

Figure 2. PD improves crypt regeneration and alters proliferation kinetics. Mice were pretreated with vehicle or PD and subjected to 15 Gy TBI. The small intestine was analyzed at the indicated times. (A) Representative images of BrdU-stained intestinal sections. Scale bar: $200 \mu$ m. (B) Quantification of BrdU $^{+}$crypt cells per cross section in A, and in mice treated with PD but no TBI (dashed black line). (C) Representative images of BrdU IHC in crypts at 0 , 4, and 24 hours. Scale bar: $20 \mu \mathrm{m}$. (D) Quantitation of BrdU+ crypt cells in C. (E) Quantification of BrdU+ CBCs at 0 hours. (F) Representative images of $\mathrm{p}-\beta$-catenin (Ser552) immunofluorescence in crypts with the indicated treatment. Dashed white lines outline the crypts. Red, $\mathrm{p}$ - $\beta$-catenin; blue, DAPI. Scale bar: $20 \mu \mathrm{m}$. (G) Quantitation of p- $\beta$-catenin ${ }^{+}$crypt cells. (B, D, E, and $\mathbf{G}$ ) Values represent the mean \pm SEM; $N=3$ mice in each group. ${ }^{*} P<0.01$ and ${ }^{* * *} P<0.001$, vehicle versus PD treatment, by unpaired, 2 -tailed Student's $t$ test. 
A

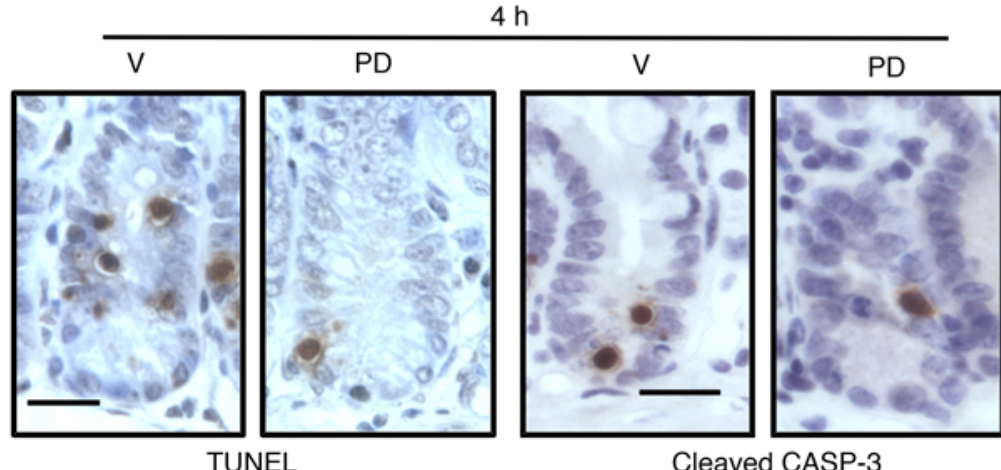

B

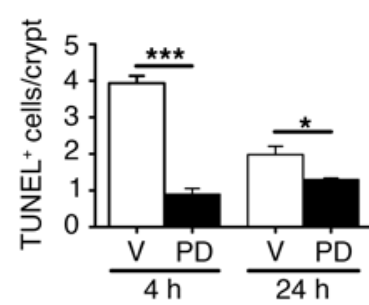

D
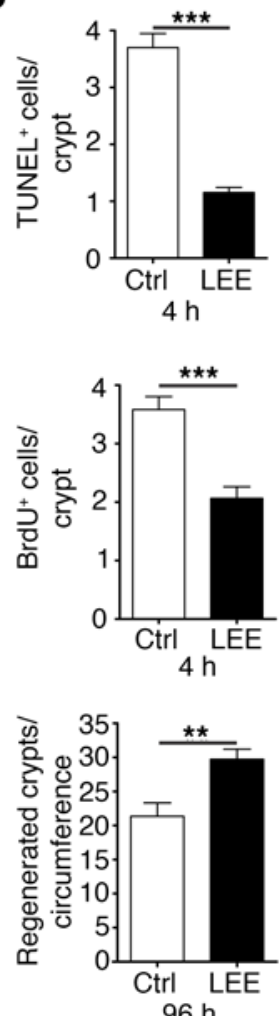

C
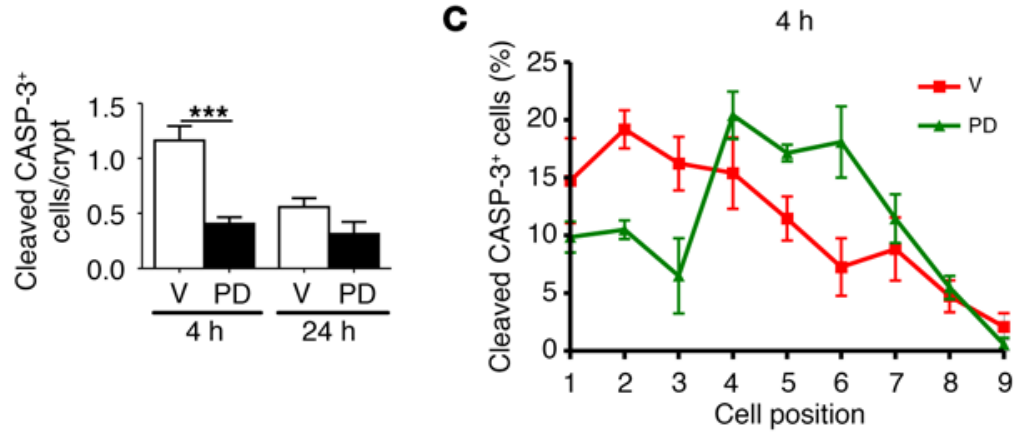

E

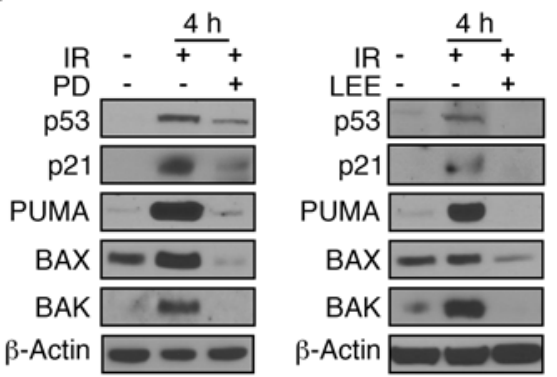

$\mathbf{F}$
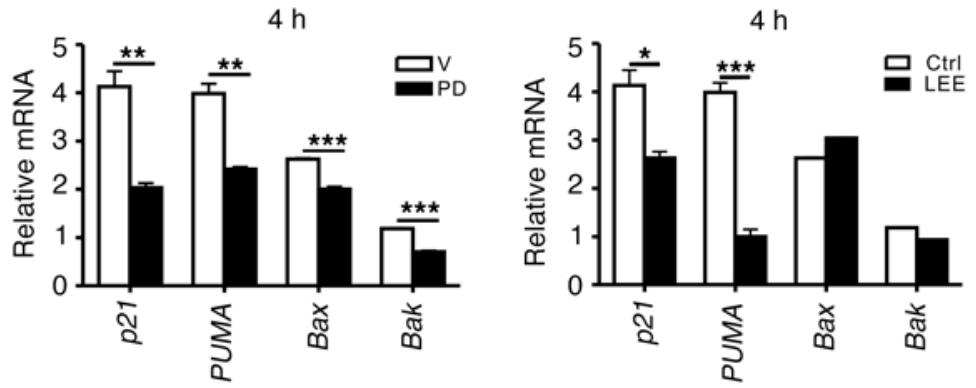

Figure 3. PD blocks IR-induced crypt apoptosis. Mice were pretreated with vehicle, PD, or LEE and subjected to 15 Gy TBI. The small intestine was analyzed at the indicated time points. (A) Representative images of TUNEL and cleaved caspase-3 (CASP-3) staining of intestinal sections at 4 hours. Scale bars: $20 \mu \mathrm{m}$. (B) Quantitation of TUNEL ${ }^{+}$and cleaved caspase- $3^{+}$crypt cells at 4 and 24 hours. (C) Fractions of cleaved caspase- $3^{+}$crypt cells at 4 hours based on cell positions. (D) Quantification of TUNEL and BrdU index at 4 hours and regenerated crypts at 96 hours in LEE-treated and control (Ctrl) mice. (E) Intestinal expression of the indicated proteins analyzed by Western blotting. $\beta$-Actin was used as the loading control. Lysates were pooled from 3 mice per group. Replicate samples run on separate gels are presented. (F) Intestinal relative expression of the indicated mRNAs versus the nontreated mice. cDNA was synthesized from RNA pooled from 3 mice per group. Similar results were obtained in at least 3 independent experiments for Western blotting and RT-PCR. (B-D and F) Values represent the mean \pm SEM; $N=3$ mice in each group. ${ }^{*} P<0.05$, ${ }^{*} P<0.01$, and ${ }^{* * *} P<0.001$, vehicle versus PD or LEE treatment, by unpaired, 2-tailed Student's $t$ test. 
7), we found that the PD group displayed better-preserved intestinal structure, a 2 fold-increase in surviving crypts, and taller villi (Figure 1, D-F). This was correlated with an approximately 2-fold increase in regenerated crypts, as measured by a microcolony assay (Figure 1, G and $\mathrm{H}$ ). Nearly all crypts were regenerated de novo following the loss of existing ones at this dose in WT mice (1, 11). PD also significantly prolonged the survival of mice after 15 Gy TBI (Supplemental Figure 1B). Therefore, PD potently prevents radiation-induced lethal GI injury.

PD treatment profoundly changes intestinal proliferation kinetics. To understand the effects of PD on crypt regeneration, we monitored proliferation kinetics using the BrdU pulse assay, which captures S-phase entry and DNA replication. In the vehicle-treated group, robust crypt proliferation decreased by nearly $90 \%$ (1,500 to 30) 72 hours after TBI, followed by the appearance of regenerated crypts by 96 hours as previously described (Figure $2 \mathrm{~A})(1,7)$. In the PD-treated group, the curve was reversed, as crypt proliferation was halted prior to radiation and gradually recovered, reaching twice that of the control group by 96 hours (Figure 2, A and B, and Supplemental Figure 2A). Interestingly, PD was very potent, reducing the fraction of $\mathrm{BrdU}^{+}$crypts from $100 \%$ to $23 \%$ prior to radiation (Supplemental Figure 2B and Supplemental Figure 2C). Exposure to 15 Gy TBI causes crypt loss between 24 and 96 hours $(1,7)$ (Supplemental Figure 2D), therefore, intestinal proliferation was quantified using 6-8 cross sections in each animal. Crypt proliferation in mice receiving PD but no TBI fully recovered within 48 hours (Figure 2B and Supplemental Figure 2E). The proliferation index of CBCs was also opposite in the control and PD groups. In the control group, the proliferation rate of $\mathrm{CBCs}$ started high and declined within 24 hours, while it started very low in the PD-treated group and increased to a significantly higher level than was observed in the controls (Figure 2, C-E). BrdU labels both ISCs and transit-amplifying (TA) cells, which greatly outnumber ISCs.

$\beta$-Catenin is an important signal transducer of the Wnt pathway, and its carboxy terminal phosphorylation (Ser552) promotes its nuclear accumulation and ISC activation, but is absent in TA cells $(23,24)$. In unirradiated mice, roughly 80 such cells were found in a cross section, or 1 cell in approximately $40 \%$ of crypts (Figure 2G and Supplemental Figure 2F) $(23,24)$. We therefore examined the kinetics of phosphorylated $\beta$-catenin ${ }^{+}\left(p-\beta\right.$-catenin $\left.{ }^{+}\right)$ cells at 4, 24, and 96 hours after radiation, key time points corresponding to ISC inactivation, activation, and peak regeneration. These cells completely disappeared (from $~ 80$ to 0) 4 hours after radiation in both the $\mathrm{PD}$-treated and control groups, indicating that ISCs were fully inactivated. However, in the PD group, their number was $80 \%$ lower 24 hours and $200 \%$ higher 96 hours after radiation compared with numbers in the control (Figure 2, F and G, and Supplemental Figure 2F). These data demonstrate that PD profoundly alters intestinal proliferation kinetics in response to radiation, which is associated with suppressed ISC activation and improved regeneration.

PD blocks IR-induced apoptosis and p53 elevation. The increase in regenerated crypts suggests that CDKi treatment enhances stem cell survival. We therefore examined crypt apoptosis by TUNEL and cleaved caspase- 3 staining 4 and 24 hours after radiation. In the PD-treated group, the number of TUNEL ${ }^{+}$or cleaved caspase $-3^{+}$crypt cells decreased dramatically compared with the numbers in the control group (Figure 3, A and B). Interestingly, the few cleaved caspase- $3^{+}$crypt cells in the PD-treated mice were found mostly above the CBCs (Figure 3C). To rule out the possibility that these protective effects were PD specific, we used another selective CDK4/6 inhibitor, LEE, which is in the late stages of clinical development (Supplemental Figure 1A). LEE, which was administered on the same schedule, also reduced crypt apoptosis and proliferation and increased crypt regeneration (Figure 3D and Supplemental Figure 3, A-C). p53/PUMA is the major mediator of IR-induced crypt apoptosis (11). We found that PD or LEE treatment strongly inhibited the accumulation of p53 and its targets such as $p 21$, PUMA, Bax, and Bak within 4 hours (Figure 3E). Realtime RT-PCR confirmed reduced mRNA expression of p53 targets (Figure 3F), but not $p 53$ (Supplemental Figure 3D). The levels of antiapoptotic BCL-2 members were not consistently affected by IR or PD (data not shown). PD treatment also suppressed IRinduced phosphorylation of JNK, ERK, and GSK3 and increased the phosphorylation of NF- $\mathrm{BB}$ and p38 at 4 hours (Supplemental Figure 3E). These data demonstrate that CDKs profoundly suppress IR-induced p53 activation and apoptosis and influence the stress responses in intestinal crypts.

PD protects LGR5 stem cells. We then used Lgr5-EGFP-IRESCreERT2 mice (25) to examine the effects of PD on ISCs. Radiation induced a greater than $85 \%$ loss of LGR 5 cells by 48 hours, which was significantly suppressed in the PD group (Figure 4, A and B, and Supplemental Figure 4A). TUNEL and GFP (LGR5) double staining indicated extensive apoptosis of LGR5 cells 4 and 24 hours after radiation, which was suppressed in the $\mathrm{PD}$-treated group (Figure 4, C and D). Therefore, LGR5 ${ }^{+} \mathrm{CBCs}$ were radiosensitive, not resistant (26), while some LGR5 +4 cells were resistant (Figure 4A). The increased cell death we observed in the PD-treated group at 24 hours was likely caused by mitotic cell death as a result of rapid recovery of intestinal proliferation (Figure 2, B and C) with unrepaired DNA or DNA damage accumulation. We then analyzed the contribution of LGR 5 cells to crypt regeneration after IR by lineage tracing in Lgr5-EGFP-ERT2 Rosa-LacZ reporter mice with a single injection of tamoxifen 18 hours before radiation. The fraction of $\mathrm{LacZ}^{+}$crypts increased by over 2-fold in the PD-treated group (from $16 \%$ to $33 \%$ ) (Figure 4, E and F). The reporter (LGR5-GFP) is expressed in $30 \%$ to $35 \%$ of crypts (25), suggesting that nearly all crypts were from LGR $5^{+}$cells in the PD-treated group. Coupled with a greater than 2-fold (from 15 to 35; Figure 1H) increase in regenerated crypts, the LGR5-specific contribution in the PD group was calculated to be 4.8 -fold higher than that seen in the control group. Using Olfm4 RNA ISH (27), we confirmed a strong protection by $\mathrm{PD}$ against the loss of $\mathrm{Olfm} 4^{+}$cells and crypts at 48 hours (Figure 4, G and H, and Supplemental Figure 4B). The location-specific Olfm 4 single-molecule signals also indicated a greater than 2-fold protection, reducing the loss from over 85\% to 30\% (area) (Supplemental Figure 4, C and D). These results indicate that LGR5 stem cells are the major target of $\mathrm{PD}$-mediated intestinal protection.

PD prevents DNA damage accumulation. Our previous work established that DNA damage at 24 hours strongly correlates with crypt regeneration after 15 Gy TBI (14). To examine the potential role of DNA damage and repair in PD-enhanced crypt regeneration, we first detected DNA DSBs by $\gamma \mathrm{H} 2 \mathrm{AX}$ staining. The levels of $\gamma \mathrm{H}_{2} \mathrm{AX}^{+}$cells were similar in the PD and control groups until the 
A

Un
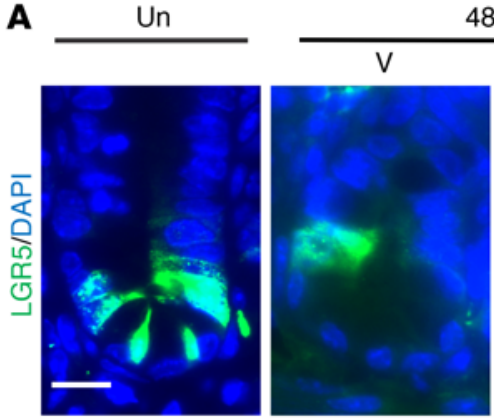

D

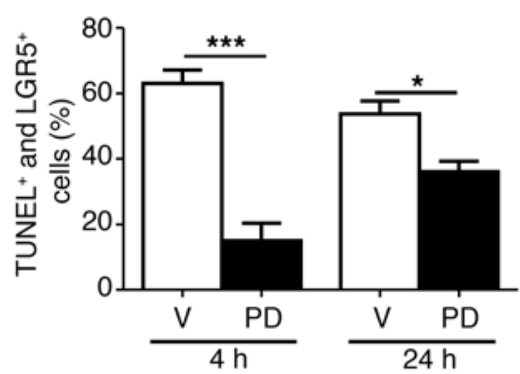

B

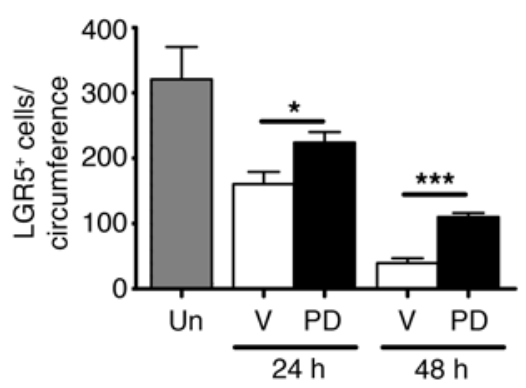

E

C

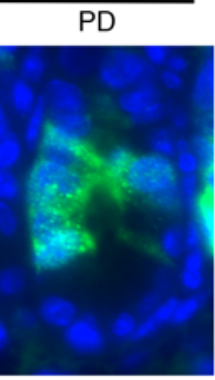

$96 \mathrm{~h}$
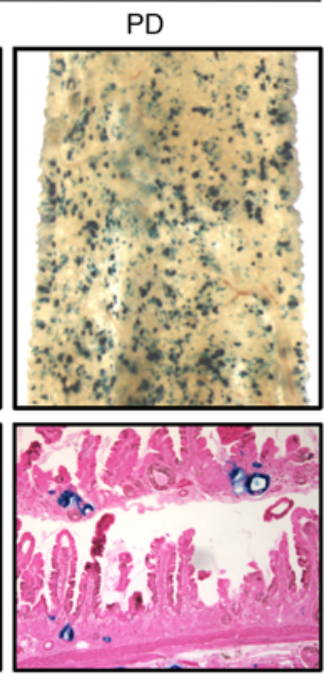
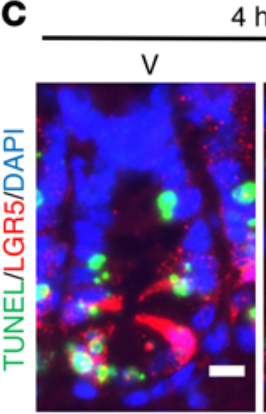

F

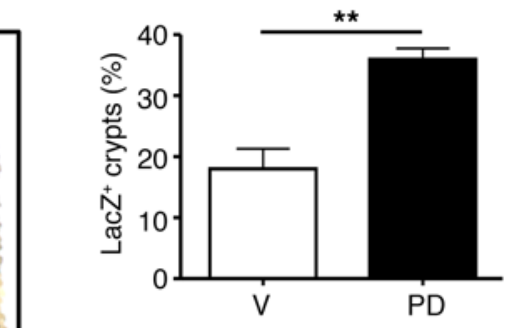

G

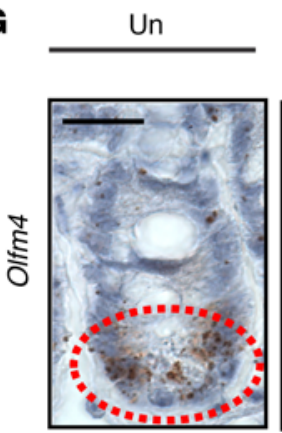

్్
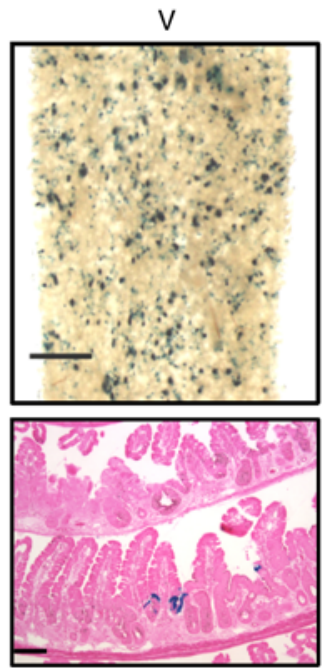

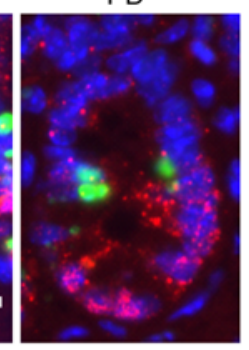

Figure 4. PD protects LGR5 ${ }^{+}$stem cells. Lgr5-EGFP or lineage-marking Lgr5-EGFP Rosa ${ }^{B /+}$ mice were pretreated with vehicle or PD and subjected to 15 Gy TBI. The small intestine was analyzed at the indicated time points. (A) Representative images of GFP/LGR5 immunofluorescence in the crypts at 48 hours. Green, GFP; blue, DAPI. Scale bar: $20 \mu \mathrm{m}$. (B) Quantitation of LGR5+ crypt cells in cross sections at 24 and 48 hours. (C) Representative images of LGR5-GFP/TUNEL double immunofluorescence in the crypts at 4 hours. Green, TUNEL; red, LGR5-GFP; blue, DAPI. Scale bar: $10 \mu \mathrm{m}$. (D) Quantification of TUNEL ${ }^{+}$and LGR5 ${ }^{+}$ stem cells (\%) in the crypts at 4 and 24 hours. (E) Representative whole-mount images and sections of X-gal-stained intestine at 96 hours. Tamoxifen was administered to Lgr5-EGFP Rosa ${ }^{B /+}$ mice once 18 hours prior to TBI. (F) Quantification of LacZ+ crypts (\%) in the intestinal sections. (G) Representative images of Olfm $4^{+}$crypt cells detected by RNAscope at 48 hours. The dotted area and arrows indicate Olfm4 signals. (H) Quantification of OLFM4 ${ }^{+}$crypt cells at 24 and 48 hours. (B, D, F, and $\mathbf{H}$ ) Values represent the mean \pm SEM; $N=3$ mice per group. ${ }^{*} P<0.05,{ }^{*} P<0.01$, and ${ }^{* * *} P<0.001$, for vehicle versus PD treatment, by unpaired, 2-tailed Student's $t$ test.

4-hour point, but were significantly lower in the $\mathrm{PD}$-treated group at 24 hours (Supplemental Figure 5A), consistent with decreased DNA damage accumulation as indicated by $\gamma \mathrm{H} 2 \mathrm{AX}$ and 53BP1 foci (Figure 5A and Supplemental Figure 5B). Inhibition of CDKs or $\mathrm{G}_{1}$ arrest can favor nonhomologous end joining (NHEJ) by inhibiting homologous recombination-related (HR-related) targets such as checkpoint kinase 1 (CHK1) and breast cancer 1 (BRCA1) in model organisms (20). The formation of BRCA1 and RAD51 foci, but not DNA-dependent protein kinase catalytic subunit (DNA-PKcs) foci, in crypt cells was strongly suppressed in the PD-treated group at 4 hours, while it was similar in the $\mathrm{CBC}(+1-4)$ and TA zones (+5-9) (Supplemental Figure 5D). Because of significant CBC loss 
A
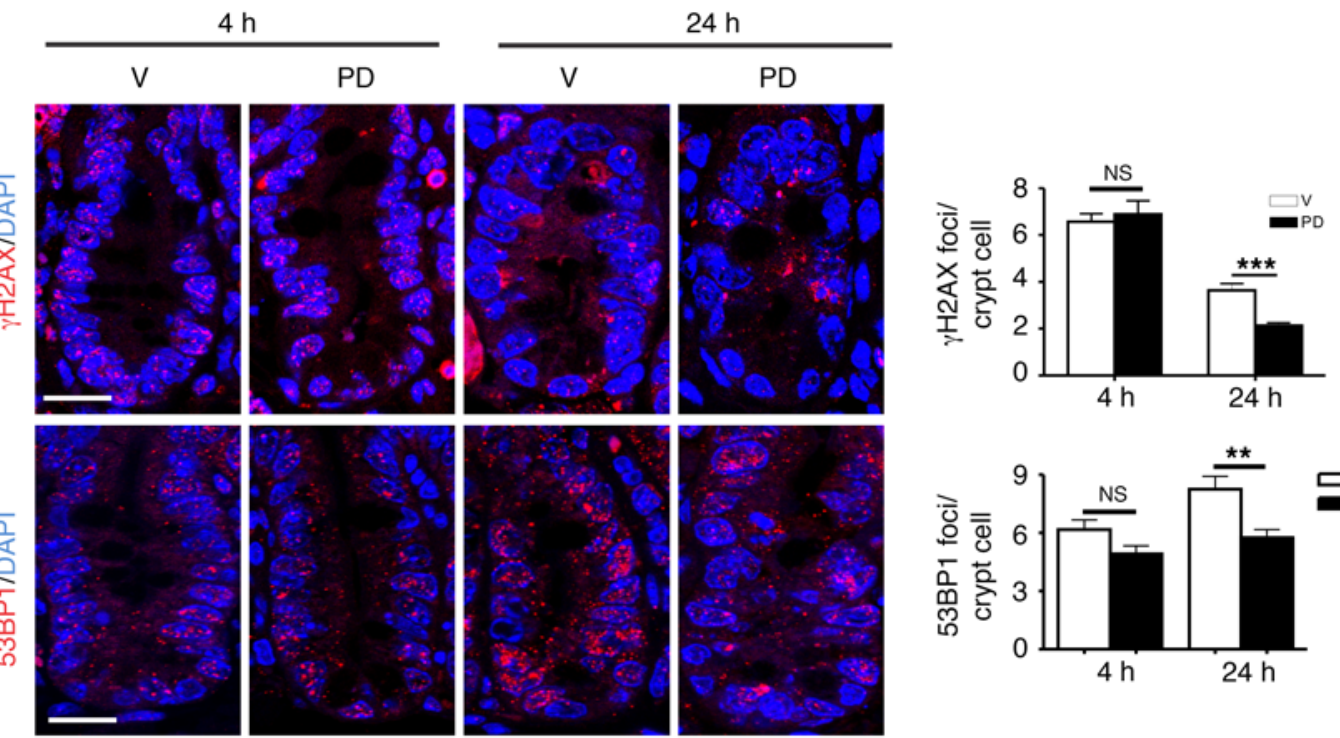

B

$4 \mathrm{~h}$
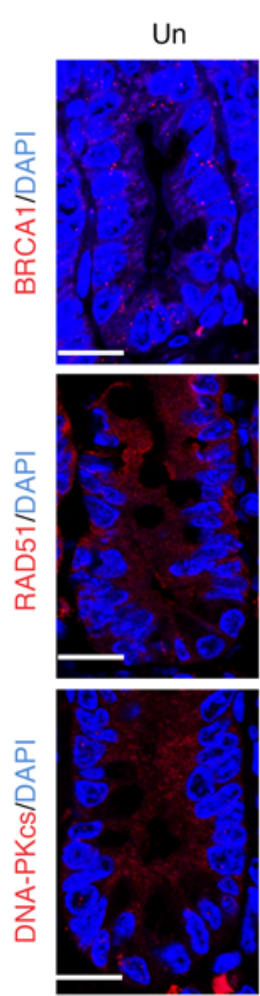

C

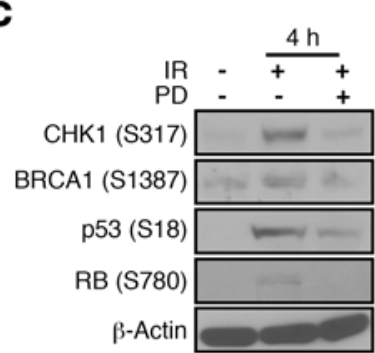

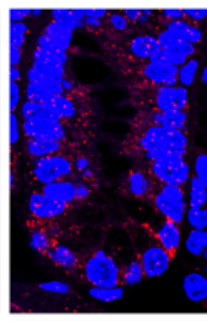
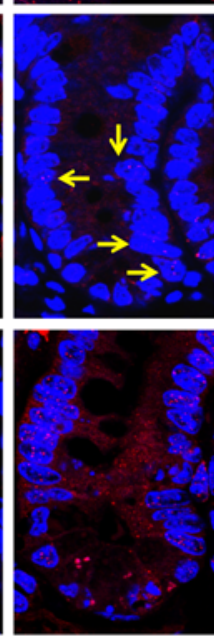

D

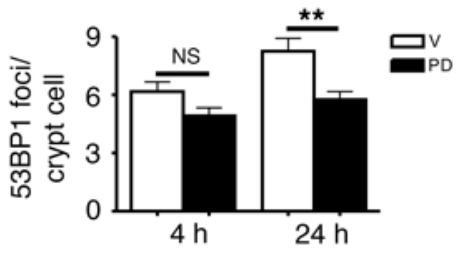

$24 \mathrm{~h}$
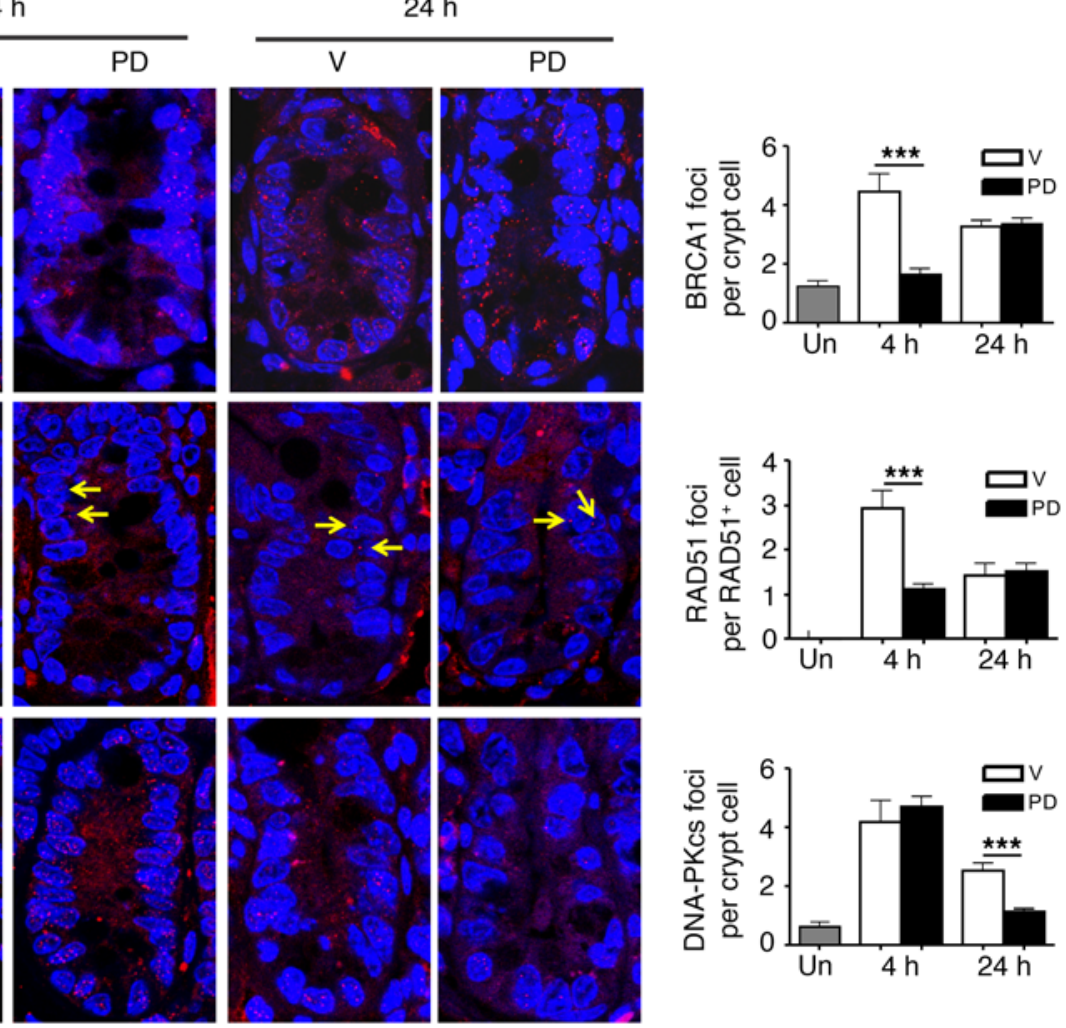
Figure 5. PD prevents DNA damage accumulation. Mice were pretreated with vehicle or PD and subjected to 15 Gy TBI. The small intestine was analyzed at the indicated time points. (A) Representative confocal images of $\gamma \mathrm{H} 2 \mathrm{AX}$ and 53BP1 (red) staining in the crypts. Blue, DAPI. Scale bars: $10 \mu \mathrm{m}$. Graph shows the quantification of foci in crypt cells. (B) Representative confocal images of BRCA1, RAD51, and DNA-PKcs (red) staining in the crypts; blue, DAPI. Scale bars: $10 \mu \mathrm{m}$. Graph shows the quantification of the respective foci in crypt cells. The arrows indicate RAD51+ foci cells, and the foci counts were calculated from RAD51 ${ }^{+}$crypt cells. (C and $\left.\mathbf{D}\right)$ Intestinal expression of the indicated proteins was analyzed by Western blotting. $\beta$-Actin was used as the loading control. Lysates were pooled from 3 mice in each group. Replicate samples run on separate gels are presented. Similar results were obtained in at least 3 independent experiments. (A and B) Values represent the mean \pm SEM; $N=3$ mice in each group. ${ }^{* *} P<0.01$ and ${ }^{* * *} P<0.001$, for vehicle versus PD treatment, by unpaired, 2-tailed Student's $t$ test.

by 24 hours (Figure $4 \mathrm{~A}$ ), we were unable to determine whether CBCs repair DNA better than TA cells do (26). The levels of BRCA1 and RAD51 foci were comparable at 24 hours in the PD and control groups, while the levels of DNA-PKcs foci were reduced in the PD group (Figure 5B and Supplemental Figure 5C). Western blotting revealed a marked inhibition of IR-induced phosphorylation of CHK1, BRCA1, and p53, with on-target inhibition of p-RB (28) in the mucosa of PD-treated mice (Figure 5C). Loss of p-CHK1 probably impairs the activation of $\mathrm{p} 53$ (S18, mouse) and retinoblastoma-binding protein 8 , endonuclease (CtIP), thus inhibiting apoptosis and the HR pathway (20), respectively. These findings provide evidence that $\mathrm{CDK}$ inhibition leads to DDR tolerance, at least in part, by preventing DNA damage accumulation with NHEJ repair pathway bias in vivo, in addition to enhanced cell survival.

$P D$-mediated intestinal protection is modulated by the p53 pathway. Suppression of $\mathrm{p} 53$ activation by PD was transient, and the levels of p53 and the p53 targets PUMA and p21 recovered within 4 hours of the last treatment (Figure 5D). The recovery of p-AKT was slightly blunted (Figure 5D). Our earlier work established that p53-dependent PUMA and p21 induction after TBI regulates crypt apoptosis and DNA damage accumulation independently. p21 is essential for reducing crypt proliferation at 4 hours and prevents the nonapoptotic cell death associated with massive replication stress and aberrant mitosis during crypt regeneration from 24 to 96 hours in both WT and PUMA-KO mice (14). We further used PUMA-KO, $p 21-\mathrm{KO}$, and $p 53-\mathrm{KO}$ mice to determine the role of the p53 pathway in $\mathrm{PD}$-mediated intestinal radioprotection.

Compared with WT control mice, the PD and PUMA-KO combination yielded a nearly 4 -fold (22.8 to 87.8 ) increase in crypt survival at 96 hours (Figure 6A and Supplemental Figure 6A), which was associated with a greater than $80 \%$ reduction of proliferation (900 to 150) and a 20-fold reduction of crypt apoptosis (4 to 0.2) at 4 hours (Figure 6B, Supplemental Figure 6B, and Supplemental Figure $6 \mathrm{C}$ ). In contrast, $p 21$ loss completely abrogated PD-mediated crypt survival at 96 hours (Figure 6A and Supplemental Figure 6A), despite suppression of crypt apoptosis and proliferation at 4 hours (Figure 6B, Supplemental Figure 6B, and Supplemental Figure 6C). $\mathrm{PD}$ also suppressed premature crypt regeneration at 72 hours in $p 21$ KO mice (Supplemental Figure 7A), however, it completely failed to suppress abnormal mitosis $\left(\gamma \mathrm{H} 2 \mathrm{AX}+/ p-\mathrm{H}^{+}\right.$cells $)$, replication stress (persistent 53BP1 foci) (14), and nonapoptotic cell death (TUNEL ${ }^{+} /$ cleaved caspase-3) (Figure 6, C and D, and Supplemental Figure 7B). Further, the benefit of PD treatment for crypt survival was also completely lost in $p 53$-KO mice, like in $p 21$-KO mice (Figure 6A), despite an inhibition of proliferation that was equally as efficient as that in $p 21-\mathrm{KO}$ and WT mice, a further decrease in apoptosis, as was observed in PUMA-KO mice 4 hours after radiation (Figure 6B, Supplemental Figure 6B and Supplemental Figure 6C), and an induced quiescence prior to radiation (Supplemental Figure 6D). $\mathrm{PD}$ treatment corrected the radioresistant DNA synthesis observed in $p 53-\mathrm{KO}$ and $p 21-\mathrm{KO}$ crypts $(11,14)$ (Figure $6 \mathrm{~B}$ and Supplemental Figure 6B). These results strongly support the idea that PD elicits a p53- and p21-dependent synergy to preserve the ISC quantity and quality necessary for intestinal recovery from radiation by suppressing PUMA-dependent apoptosis and DNA damage accumulation beyond the cell-cycle kinetics, per se.

\section{Discussion}

Radiation remains one of the most widely used cancer treatments, while normal tissue toxicities are often dose limiting and can lead to long-term complications. There is currently no FDA-approved agent that prevents or treats radiotherapy-induced GI toxicity (8, 9). The CDKi PD has recently been approved by the FDA for cancer treatment, and more agents are in development to target the overactive $G_{1} / S$ transition, which is the commitment step to DNA replication. In this study, we demonstrate that CDKi strongly protects against IR-induced lethal intestinal injury by inducing transient stem cell quiescence and p53 shutdown to improve stem cell quantity and quality. This is consistent with the idea that blocking p53-dependent apoptosis, but not p53-dependent protective $\mathrm{DDR}$, is required to maximize intestinal radioprotection $(6,11,14)$. Our findings provide in vivo evidence that CDKs play a critical role in regulating genome stability and stem cell fate in a p53-dependent manner. Induction of stem cell quiescence might therefore be a highly selective way for normal tissue protection, but not for cancer cells that have lost p53- or RB-dependent cell-cycle control.

DDR is composed of elaborate signaling networks to sense different types of DNA damage and coordinate a series of responses to preserve genome stability. These responses include activation of cell-cycle checkpoints, DNA repair, senescence, and cell death (19) and critically control cell type-specific outcomes (29). Cell quiescence has long been known to protect hematopoietic stem cells (HSCs) against cytotoxic agents (30-32), while the underlying mechanisms remain largely unknown. Cell-cycle modulators also protected normal cells against chemotherapy (33). More recently, 2 groups reported that selective $\mathrm{CDK} 4 / 6$ inhibitors protect and, to a lesser extent, mitigate the hematopoietic toxicity induced by radiotherapy or chemotherapy in mice (34-36). Another group reported that fasting protects the small intestine from chemotherapy, with reduced apoptosis and faster resolution of DSBs (37). Consistent with these findings, our work identifies 2 prosurvival DDR mechanisms for induced quiescence via transient and perhaps selective modulation of DDR in normal cells, as discussed below.

First, our study demonstrates that induced quiescence leads to transient but nearly complete loss of p53-dependent PUMA induction and ISC apoptosis, whereas p21 or timely recovery of p21 is 
A

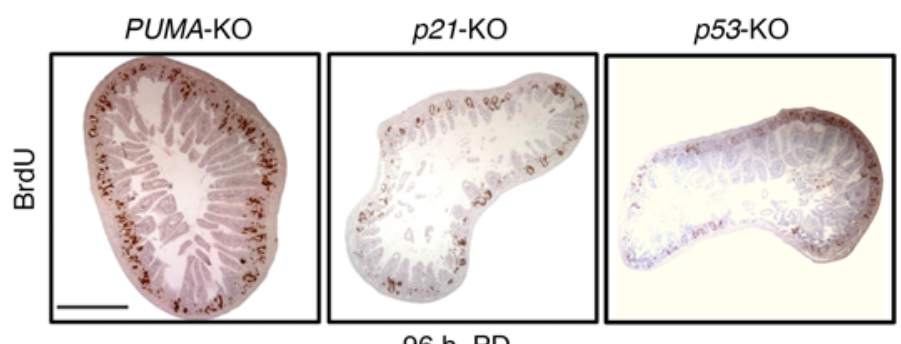

$96 \mathrm{~h}, \mathrm{PD}$

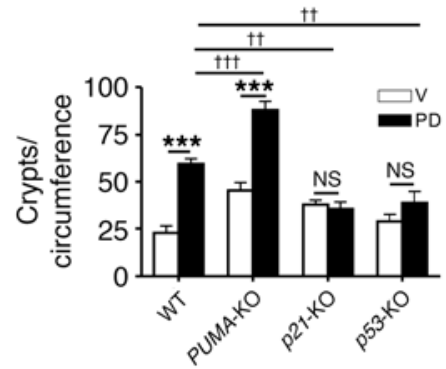

B

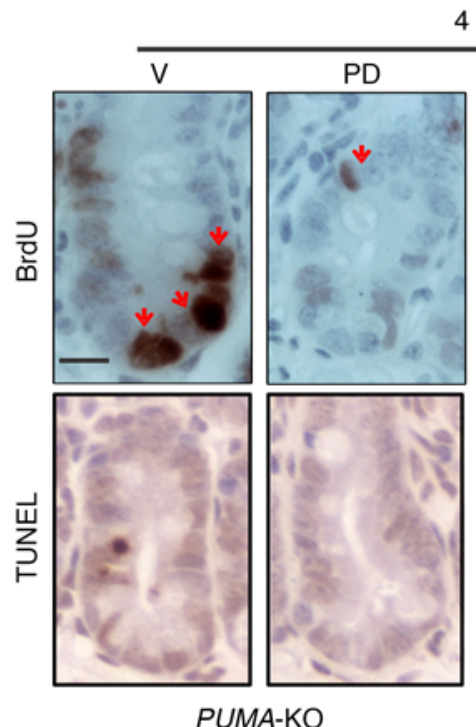

$4 \mathrm{~h}$
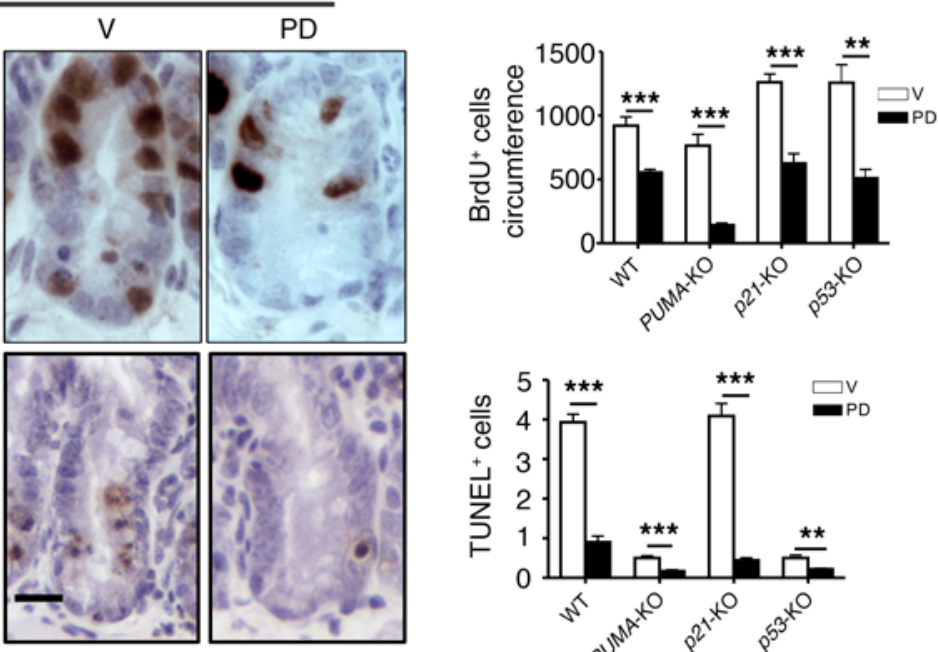

C

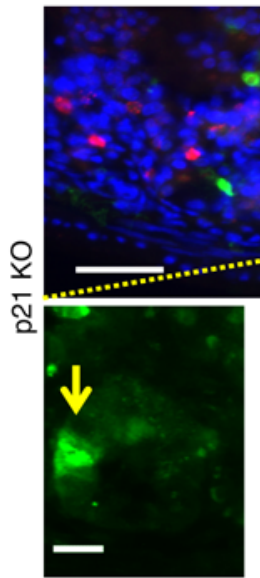

$\gamma \mathrm{H} 2 \mathrm{AX}$

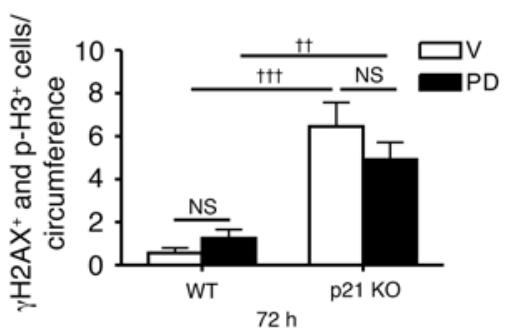

D

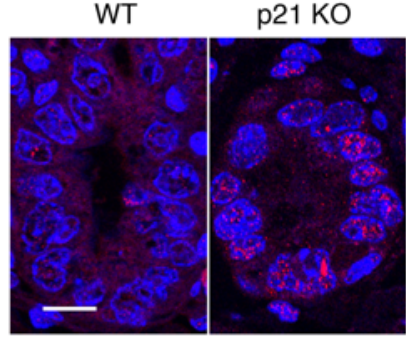

53BP1/DAPI

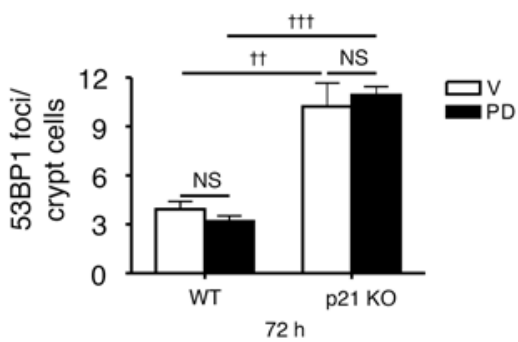

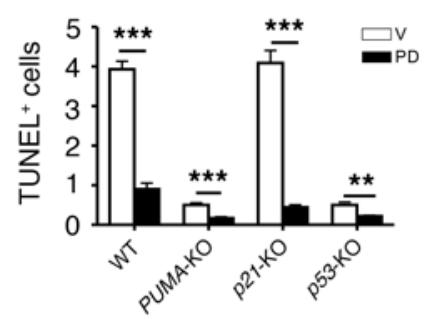


Figure 6. Intestinal radioprotection by PD requires the $\mathbf{p} 53$ pathway. Mice of the indicated genotypes were pretreated with vehicle or PD and subjected to 15 Gy TBI. The small intestine was analyzed at the indicated time points. (A) Representative images of BrdU IHC in PUMA-KO, p21-KO, and p53-KO crypts at 96 hours. Scale bar: $500 \mu \mathrm{m}$. Graph shows the quantification of surviving crypts at 96 hours in mice of the 4 genotypes. (B) Representative images of BrdU and TUNEL staining at 4 hours. Scale bars: $10 \mu \mathrm{m}$. Graph shows the quantification of $\mathrm{BrdU}^{+}$and TUNEL $\mathrm{L}^{+}$crypt cells from mice of the 4 genotypes at 4 hours. (C) Top: Representative image of $\gamma \mathrm{H} 2 \mathrm{AX} / \mathrm{p}-\mathrm{H} 3$ double immunofluorescence in $p 21-\mathrm{KO}$ crypts with higher-magnification images shown below. Green, $\gamma \mathrm{H} 2 \mathrm{AX}$; red, $\mathrm{p}-\mathrm{H} 3$; blue, DAPI. Scale bar: $50 \mu \mathrm{m}, 20 \mu \mathrm{m}$ (higher-magnification images). Graph shows the quantification of $\gamma \mathrm{H} 2 \mathrm{AX} / \mathrm{p}-\mathrm{H} 3$ double-positive crypt cells at 72 hours. (D) Representative images of 53BP1 immunofluorescence (red) in the crypts at 72 hours; blue, DAPI. Scale bar: $10 \mu \mathrm{m}$. Graph shows the quantification of 53BP1 foci at 72 hours. Values represent the mean $\pm \mathrm{SEM} ; N=3$ mice in each group. ${ }^{* *} P<0.01$ and ${ }^{* * *} P<0.001$, for vehicle versus PD treatment in mice of the same genotype, by unpaired, 2-tailed Student's $t$ test; ${ }^{{ }^{\dagger \dagger}} P<0.01$ and ${ }^{\text {t+t}} P<0.001$, for WT versus the indicated genotypes or treatment, by 1-way ANOVA followed by Bonferroni's multiple comparisons test.

critical for preventing DNA damage accumulation during crypt and stem cell regeneration. DDR tolerance is important for cell and organism survival and mutagenesis (38). Interestingly, early studies have demonstrated that distinct domains of p21 bind to CDKs and proliferating cell nuclear antigen (PCNA) to regulate the cell cycle and DNA replication and repair, respectively $(39,40)$. PCNA binding improves the accuracy of translesion DNA synthesis (TLS) by decreasing the interactions of PCNA and low-fidelity TLS polymerases $(41,42)$. Therefore, cell-cycle-dependent and -independent mechanisms likely work together to increase survival and repair damage before or during proliferation to avoid mitotic catastrophe.

Second, our study provides strong evidence that CDK activity influences the choice of DNA repair pathways in vivo. HR and NHEJ are 2 major pathways for repairing DSBs and are differentially utilized during the cell cycle $(20,43)$. Suppressed HR upon induced quiescence likely leads to increased NHEJ dependence and involves several mechanisms, such as lack of HR repair templates due to $G_{1}$ arrest and loss of CHK1-mediated CtIP-dependent DNA end resection and CDK-mediated p-BRCA1 (Ser1497). The importance of NHEJ in repairing IR-induced DNA lesions in the intestine is further supported by the observations that deficiency in 53BP1 $(44,45)$, poly(ADP-ribose) polymerase 1 (PARP1) (46), or DNA-PKcs (47) exacerbates crypt loss and tissue injury. These deficiencies are predicted to abrogate or attenuate the intestinal protection afforded by PD. Mutation in $B R C A 1 / 2(48,49)$ or compromised CDK1 in BRCA-proficient cancer cells (50) leads to defective HR and increased sensitivity to PARP inhibitors (PARPi). It is therefore tempting to speculate that CDKi induce a transient state of "BRCA-lessness" and NHEJ dependence or PARPi sensitivity and that $p 53$ status plays a potential role in modulating patients' responses to CDKi/PARPi with DNA-damaging agents. However, the role and underlying mechanisms of increased NHEJ dependence in CDKi-mediated intestinal radioprotection need to be directly tested using mouse and cell models.

In addition, our study reveals a key role of LGR5 stem cells in IR-induced acute intestinal injury and regeneration, focusing on cell-intrinsic DDR using the well-characterized single, high-dose TBI model $(1,7,22)$. Stem cells are regulated by extracellular cues from the niche (51), while very little is known about how the niche regulates intestinal recovery from injury. The surprisingly potent synergic protection observed in the PD and PUMA-KO combination strongly suggests that the extent of ISC loss connects nichebased programs with tissue regeneration and stem cell activation, perhaps via damage-associated molecular patterns (DAMPs) (7) released from dying cells. However, our study cannot rule out the potential effects of CDKi in other cells.
Successful cancer treatment by radiation and chemotherapy relies on the selective killing of cancer cells, with adequate preservation of the normal tissue (52). The p53 and RB pathways are frequently deregulated in human cancer (53), leading to deregulated CDK $4 / 6$ activity and control of the $G_{1} / S$ transition, both of which are being targeted by $\operatorname{CDKi}(15,16)$. It is important to mention that CDKi are different from other radioprotectors such as antioxidants or growth factors, which can either protect both normal and cancers cells or exacerbate tissue injury after repeated exposures due to an accelerated cell cycle (7). Our work supports the finding that reversible CDKi induce protective DDR selectively in normal tissues with WT p53, but not in cancers with deregulated p53 (35), and clearly warrants further experimentation.

\section{Methods}

Mice and treatment. Eight- to ten-week-old $P U M A^{-1-}$ (PUMA-KO), $p 21^{-/-}(p 21-\mathrm{KO})$, and $p 53^{-/-}(p 53-\mathrm{KO})$ mice were generated from homozygous breeding. The generation and use of PUMA-KO (11), p21-KO (14), p53-KO (JAX 002101), and Lgr5-EGFP (Lgr5-EGFPIRES-CreER ${ }^{T 2}$ ) (25) mice have been previously described. Lgr5-EGFP

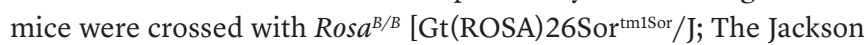
Laboratory] to generate Lgr5-EGFP Rosa ${ }^{B /+}$ (LacZ reporter) mice. All strains were on or had been backcrossed with mice on a C57BL/6 background for more than 10 generations (F10). For TBI, mice were irradiated at a rate of $76 \mathrm{cGy} / \mathrm{min}$ in a ${ }^{137} \mathrm{Cs}$ irradiator (Mark I; JL Shepherd and Associates). ABI was administered in the form of $\mathrm{x}$-ray with a clinical-grade linear accelerator (Varian Medical Systems) as described previously (22). For CDKi, PD (CT-PD2991; Chemietek) (30 $\mathrm{mg} / \mathrm{ml}$ in sodium lactate buffer, $\mathrm{pH} 4$.0) was given by oral gavage at a dose of $150 \mathrm{mg} / \mathrm{kg} \mathrm{BW}$. LEE (CT-LEE011; Chemietek) was dissolved in 0.5\% methylcellulose (M0512; Sigma-Aldrich) and administered by oral gavage at a dose of $150 \mathrm{mg} / \mathrm{kg}$.

Analysis of $m R N A$ and protein expression. Intestinal mucosa scraping and isolation of total RNA and protein extracts from mouse tissues were performed as previously described $(54,55)$. Real-time RT-PCR was performed on a CFX96 Touch Real-Time PCR Detection System (Bio-Rad) with SYBR Green (Invitrogen, Thermo Fisher Scientific). Primers are listed in Supplemental Table 1. Total protein extracts were subjected to NuPAGE gel electrophoresis (Invitrogen, Thermo Fisher Scientific), followed by Western blotting. Details on the antibodies used are provided in the Supplemental Methods.

Tissue processing, histological analysis, and TUNEL and BrdU staining. BrdU (Sigma Chemical Co.) was administered by i.p. injection to all mice 2 hours prior to sacrifice at a dose of $100 \mathrm{mg} / \mathrm{kg}$. Tissues were harvested, fixed, and embedded in paraffin as described previously $(11,22)$. Sections $(5-\mu \mathrm{m})$ were subjected to H\&E stain- 
ing for histological analysis. Detailed information can be found in the Supplemental Methods.

IHC and immunofluorescence. Prior to immunostaining, paraffin-embedded sections were subjected to deparaffinization and antigen retrieval (boiling sections for 10 minutes in $0.1 \mathrm{M}$ citrate buffer, pH 6.0, with $1 \mathrm{mM}$ EDTA). Detailed information can be found in the Supplemental Methods.

RNAscope ISH assay. Murine Olfm4 probe (catalog 311831) and RNAscope 2.0 HD Reagent Kit-BROWN (catalog 310035) were purchased from Advanced Cell Diagnostics (ACD) and used according to the manufacturer's instructions. In brief, deparaffinized 5 - $\mu$ m paraffin sections were pretreated with Pretreat 1, 2, and 3 (ACD). Olfm 4 was added to the slides and incubated in the HybEZ oven (catalog 310010; ACD) for 2 hours at $40^{\circ} \mathrm{C}$. After a 6-step signal amplification, tissues were detected by DAB and counterstained. Slides were mounted with Crystal Mount (M02; Biomeda) and photographed.

$X$-Gal staining for $\beta$-gal. $\beta$-Gal tissue whole-mount staining was performed as previously described (25). Detailed methods are found in supplemental materials.

Statistics. GraphPad Prism 4 (GraphPad Software) was used for statistical analyses. Survival was analyzed by the log-rank test. Data were analyzed by an unpaired, 2-tailed Student's $t$ test or ANOVA, followed by Turkey's or Bonferroni's test, in which multiple comparisons were performed using the method of least significant difference. A $P$ value of less than 0.05 was considered significant.
Study approval. The procedures for all animal experiments were reviewed and approved by the IACUC of the University of Pittsburgh.

\section{Author contributions}

LW and JY designed the experiments. LW, XW, BJL, and ME performed the experiments. LW and JY analyzed the data. LZ and JG provided critical reagents and read the manuscript. LW and JY wrote the manuscript. JY conceived the study and supervised all experiments.

\section{Acknowledgments}

We thank the members of the Yu and Zhang laboratories and Ben Van Houten and Christopher Bakkenist (University of Pittsburgh, Pittsburgh, Pennsylvania, USA) for their helpful discussions. This work was supported in part by the American Cancer Society grant RGS-10-124-01-CCE (to JY); NIH grants U01-DK085570 (to JY); U19-A1068021 (to JG); and CA106348 (to LZ). This project used the University of Pittsburgh Cancer Institute shared glassware, animal, and cell and tissue imaging facilities that were supported in part by National Cancer Institute (NCI) award P30CA047904.

Address correspondence to: Jian Yu, Hillman Cancer Center Research Pavilion, Suite 2.26h, 5117 Centre Avenue, Pittsburgh, Pennsylvania 15213, USA. Phone: 412.623.7786; E-mail: yuj2@upmc.edu.
1. Potten CS. Radiation, the ideal cytotoxic agent for studying the cell biology of tissues such as the small intestine. Radiat Res. 2004;161(2):123-136.

2. Bjerknes M, Cheng H. Clonal analysis of mouse intestinal epithelial progenitors. Gastroenterology. 1999;116(1):7-14.

3. Barker N, van Oudenaarden A, Clevers H. Identifying the stem cell of the intestinal crypt: strategies and pitfalls. Cell Stem Cell. 2012;11(4):452-460.

4. Metcalfe C, Kljavin NM, Ybarra R, de Sauvage FJ. Lgr5+ stem cells are indispensable for radiation-induced intestinal regeneration. Cell Stem Cell. 2014;14(2):149-159.

5. Qiu W, Leibowitz B, Zhang L, Yu J. Growth factors protect intestinal stem cells from radiation-induced apoptosis by suppressing PUMA through the PI3K/AKT/p53 axis. Oncogene. 2010;29(11):1622-1632.

6. Wang X, et al. Pharmacologically blocking p53-dependent apoptosis protects intestinal stem cells and mice from radiation. Sci Rep. 2015;5:8566.

7. Yu J. Intestinal stem cell injury and protection during cancer therapy. Transl Cancer Res. 2013;2(5):384-396.

8. Greenberger JS. Radioprotection. In Vivo. 2009;23(2):323-336

9. Hauer-Jensen M, Denham JW, Andreyev HJ. Radiation enteropathy--pathogenesis, treatment and prevention. Nat Rev Gastroenterol Hepatol. 2014;11(8):470-479.

10. Merritt AJ, et al. The role of p53 in spontaneous and radiation-induced apoptosis in the gastrointestinal tract of normal and p53-deficient mice. Cancer Res. 1994;54(3):614-617.
11. Qiu W, et al. PUMA regulates intestinal progenitor cell radiosensitivity and gastrointestinal syndrome. Cell Stem Cell. 2008;2(6):576-583.

12. Merritt AJ, Allen TD, Potten CS, Hickman JA. Apoptosis in small intestinal epithelial from p53null mice: evidence for a delayed, p53-independent G2/M-associated cell death after gammairradiation. Oncogene. 1997;14(23):2759-2766.

13. Komarova EA, et al. Dual effect of $\mathrm{p} 53$ on radiation sensitivity in vivo: $\mathrm{p} 53$ promotes hematopoietic injury, but protects from gastro-intestinal syndrome in mice. Oncogene. 2004;23(19):3265-3271.

14. Leibowitz BJ, Qiu W, Liu H, Cheng T, Zhang L, Yu $\mathrm{J}$. Uncoupling p53 functions in radiation-induced intestinal damage via PUMA and p21. Mol Cancer Res. 2011;9(5):616-625.

15. Sherr CJ, Roberts JM. CDK inhibitors: positive and negative regulators of G1-phase progression. Genes Dev. 1999;13(12):1501-1512.

16. Ho A, Dowdy SF. Regulation of G(1) cell-cycle progression by oncogenes and tumor suppressor genes. Curr Opin Genet Dev. 2002;12(1):47-52.

17. Fry DW, et al. Specific inhibition of cyclin-dependent kinase 4/6 by PD 0332991 and associated antitumor activity in human tumor xenografts. Mol Cancer Ther. 2004;3(11):1427-1438.

18. Bartek J, Lukas J. Pathways governing G1/S transition and their response to DNA damage. FEBS Lett. 2001;490(3):117-122.

19. Harper JW, Elledge SJ. The DNA damage response: ten years after. Mol Cell. 2007; 28(5):739-745.

20. Wohlbold L, Fisher RP. Behind the wheel and under the hood: functions of cyclin-dependent kinases in response to DNA damage. DNA Repair (Amst). 2009;8(9):1018-1024.
21. Terry NH, Travis EL. The influence of bone marrow depletion on intestinal radiation damage. Int J Radiat Oncol Biol Phys. 1989;17(3):569-573.

22. Leibowitz BJ, et al. Ionizing irradiation induces acute haematopoietic syndrome and gastrointestinal syndrome independently in mice. Nat Commun. 2014;5:3494.

23. Fevr T, Robine S, Louvard D, Huelsken J. Wnt/ beta-catenin is essential for intestinal homeostasis and maintenance of intestinal stem cells. $\mathrm{Mo}$ Cell Biol. 2007;27(21):7551-7559.

24. Qiu W, et al. Chemoprevention by nonsteroidal anti-inflammatory drugs eliminates oncogenic intestinal stem cells via SMACdependent apoptosis. Proc Natl Acad Sci US A. 2010;107(46):20027-20032.

25 . Barker $\mathrm{N}$, et al. Identification of stem cells in small intestine and colon by marker gene Lgr5. Nature. 2007;449(7165):1003-1007.

26. Hua G, et al. Crypt base columnar stem cells in small intestines of mice are radioresistant. Gastroenterology. 2012;143(5):1266-1276.

27. van der Flier LG, Haegebarth A, Stange DE, van de Wetering M, Clevers H. OLFM4 is a robust marker for stem cells in human intestine and marks a subset of colorectal cancer cells. Gastroenterology. 2009;137(1):15-17.

28. Guo J, Longshore S, Nair R, Warner BW. Retinoblastoma protein (pRb), but not p107 or p130, is required for maintenance of enterocyte quiescence and differentiation in small intestine. J Biol Chem. 2009;284(1):134-140.

29. Lane AA, Scadden DT. Stem cells and DNA damage: persist or perish? Cell. 2010;142(3):360-362.

30. Li L, Bhatia R. Stem cell quiescence. Clin Cancer Res. 2011;17(15):4936-4941. 
31. Tubiana M, Carde P, Frindel E. Ways of minimising hematopoietic damage induced by radiation and cytostatic drugs--the possible role of inhibitors. Radiother Oncol. 1993;29(1):1-17.

32. Mohrin M, et al. Hematopoietic stem cell quiescence promotes error-prone DNA repair and mutagenesis. Cell Stem Cell. 2010;7(2):174-185.

33. Blagosklonny MV, Pardee AB. Exploiting cancer cell cycling for selective protection of normal cells. Cancer Res. 2001;61(11):4301-4305.

34. Johnson SM, et al. Mitigation of hematologic radiation toxicity in mice through pharmacological quiescence induced by CDK4/6 inhibition. J Clin Invest. 2010;120(7):2528-2536.

35. Roberts PJ, et al. Multiple roles of cyclin-dependent kinase 4/6 inhibitors in cancer therapy. J Natl Cancer Inst. 2012;104(6):476-487.

36. Bisi JE, Sorrentino JA, Roberts PJ, Tavares FX, Strum JC. Preclinical characterization of G1T28: A novel CDK4/6 inhibitor for reduction of chemotherapy-induced myelosuppression. Mol Can cer Ther. 2016;15(5):783-793.

37. Tinkum KL, et al. Fasting protects mice from lethal DNA damage by promoting small intestinal epithelial stem cell survival. Proc Natl Acad Sci U S A. 2015;112(51):E7148-E7154.

38. Ghosal G, Chen J. DNA damage tolerance: a double-edged sword guarding the genome. Transl Cancer Res. 2013;2(3):107-129.

39. Li R, Waga S, Hannon GJ, Beach D, Stillman B.
Differential effects by the $\mathrm{p} 21 \mathrm{CDK}$ inhibitor on PCNA-dependent DNA replication and repair. Nature. 1994;371(6497):534-537.

40. Chen J, Jackson PK, Kirschner MW, Dutta A. Separate domains of $\mathrm{p} 21$ involved in the inhibition of Cdk kinase and PCNA. Nature. 1995;374(6520):386-388.

41. Prives C, Gottifredi V. The p 21 and PCNA partnership: a new twist for an old plot. Cell Cycle. 2008;7(24):3840-3846.

42. Soria G, Speroni J, Podhajcer OL, Prives C, Gottifredi V. p21 differentially regulates DNA replication and DNA-repair-associated processes after UV irradiation. J Cell Sci. 2008;121(Pt 19):3271-3282.

43. Cerqueira A, Santamaría D, Martínez-Pastor B, Cuadrado M, Fernández-Capetillo $\mathrm{O}$, Barbacid M. Overall Cdk activity modulates the DNA damage response in mammalian cells. J Cell Biol. 2009;187(6):773-780.

44. Clarke AR, Jones N, Pryde F, Adachi Y, Sansom OJ. 53BP1 deficiency in intestinal enterocytes does not alter the immediate response to ionizing radiation, but leads to increased nuclear area consistent with polyploidy. Oncogene. 2007;26(43):6349-6355.

45. Panier S, Boulton SJ. Double-strand break repair: 53BP1 comes into focus. Nat Rev Mol Cell Biol. 2014;15(1):7-18.

46. Ishizuka S, et al. Poly(ADP-ribose) polymerase-1 is a survival factor for radiation-exposed intestinal epithelial stem cells in vivo. Nucleic Acids Res. 2003;31(21):6198-6205.

47. Rotolo JA, et al. Regulation of ceramide synthase-mediated crypt epithelium apoptosis by DNA damage repair enzymes. Cancer Res. 2010;70(3):957-967.

48. Javle M, Curtin NJ. The role of PARP in DNA repair and its therapeutic exploitation. Br JCancer. 2011;105(8):1114-1122.

49. Lord CJ, Ashworth A. Mechanisms of resistance to therapies targeting BRCA-mutant cancers. Nat Med. 2013;19(11):1381-1388.

50. Johnson N, et al. Compromised CDK1 activity sensitizes BRCA-proficient cancers to PARP inhibition. Nat Med. 2011;17(7):875-882.

51. Li L, Clevers H. Coexistence of quiescent and active adult stem cells in mammals. Science. 2010;327(5965):542-545.

52. Gudkov AV, Komarova EA. Radioprotection: smart games with death. JClin Invest. 2010;120(7):2270-2273.

53. Hanahan D, Weinberg RA. Hallmarks of cancer: the next generation. Cell. 2011;144(5):646-674.

54. Wu B, et al. p53 independent induction of PUMA mediates intestinal apoptosis in response to ischaemia-reperfusion. Gut. 2007;56(5):645-654.

55. Qiu W, Carson-Walter EB, Kuan SF, Zhang L, Yu J. PUMA suppresses intestinal tumorigenesis in mice. Cancer Res. 2009;69(12):4999-5006. 